\title{
EDITORIAL
}

\section{METABOLIC PHENOTYPING: A NEW CONCEPT}

\author{
S Naher ${ }^{1}$, N Hoque ${ }^{1}$ \\ ${ }^{1}$ Dept of Biochemistry, Bashundhara Ad-din Medical College, South Keraniganj, Dhaka
}

Early in the $20^{\text {th }}$ century, obesity was considered as a condition associated with high socioeconomic status confined mostly in developed countries. But recent data shows a dramatic increase in incidence of obesity in developing countries making it an important public health issue ${ }^{1}$.

Of many anthropometric measurements, body mass index (BMI) is the most accepted and widely used one to assess the health risk arising out of overweight and obesity because it reflects the risk of developing type 2 diabetes mellitus, cardiovascular and many other metabolic disorders. But now using of BMI as risk assessment indicator of such disorders becomes debatable. Researchers came up with a question: Does BMI always reflects the risk of obesity related metabolic disorders ${ }^{2}$ ?-

Development of obesity related cardiometabolic disorders depend on fat weight gain, location of adipose tissue, hypertrophy, hyperplasia as well as inflammation and adipokine profile of adipose tissue. Adipose tissue plays a central role in lipid and glucose metabolism and produces a large number of hormones and cytokines. Hypertrophy or inflammation of adipose tissue, causes adipocyte dysfunction (AD) resulting in different cardiometabolic abnormalities, such as type-2 DM, hypertension, atherosclerosis, cardiovascular diseases (CVD) etc ${ }^{3}$.

Obesity related metabolic abnormalities do not, however, affect all obese equally possibly due to the magnitude of adipocyte dysfunction ${ }^{4}$. On the other hand, a subset of normal weight and overweight individuals suffer from metabolic disturbances that are characteristic of obesity, which may be due to presence of adipocyte

Bangladesh J Med Biochem 2018; 11(1): iii-iv dysfunction ${ }^{5}$. Insulin resistance, hyper insulinemia, dyslipidemia may go undetected in a sub group of normal weight individuals for years. As their normal body weight mask the need for their early detection and treatment, they remain undetected and uncared. On the other hand some individuals who are obese according to BMI classification, may be metabolically healthy with no or little risk of CVD, because they may not have adipocyte dysfunction. But as they are obese according to BMI, they are given special attention than actually needed.

To solve this issue a new term metabolic obesity (MO) came up. MO may be defined as individuals with unhealthy metabolic profile irrespective of BMI. Pajunen et al. (2011) and Goday et al. (2016) coined the term metabolically healthy and metabolically unhealthy phenotypes within different BMI group (normal weight, overweight, obese) ${ }^{6,7}$. They used the components of metabolic syndrome (MetS) (waist circumference, TG, HDL-C, FBS, $\mathrm{BP})$ for this classification. Individual having $\geqslant 3$ components abnormal is regarded as metabolically unhealthy. Wildman et al. (2008) evaluated metabolic health little bit differently by the components of cardiometabolic disabilities (CA). Individual having 2 or more abnormal components of CA out of 6 components (BP, FBS, HOMA-IR, TG, HDL-C, hsCRP) is regarded as metabolically unhealthy; whereas, presence of less than 2 components of CA regarded as metabolically healthy irrespective $\mathrm{BMI}^{8}$.

Therefore in each BMI group; normal weight (BMI 18.5-24.9), overweight (BMI 25-29.9) and obese $(\mathrm{BMI} \geqslant 30)$; metabolically healthy and unhealthy phenotypes are categorized and thus 
six metabolic phenotypes are identified. These are MHNW (metabolically healthy normal weight), MONW (metabolically obese normal weight), MHOW (metabolically healthy overweight), MOOW (metabolically obese overweight), MHO (metabolically healthy obese), MUO (metabolically unhealthy obese). There are different other methods proposed by different researchers to define metabolic obesity and to identify obesity phenotypes; but they are yet to reach a universal consensus.

So researchers in this field should come up to solve this debatable issue. Clinicians and nutritionist should also pay due attention to identify individual who is really at risk of developing complications related to overweight and obesity for better management.

\section{Reference}

1. Popkin BM, Gordon-Larsen P. The nutrition transition: worldwide obesity dynamics and their determinants. Int J Obes Relat Metab Disord 2004; 28(3): 2-9.

2. Tatiana FS, Raquel DM, Ana Paula B, Maria P. Main characteristics of metabolically obese normal weight and metabolically healthy obese phenotypes. Nutrition Review 2007; 73(3): 175-190.

3. Yamauchi T, Kamon J, Waki H. The fat derived hormone adiponectin reverses insulin resistance associated with both lipoatrophy and obesity. Nat Med 2001; 7(1): 941-946.
4. Bluher M. The distinction of metabolically healthy from unhealthy obese individuals. Nutr Metab 2010; 21(1): 38-43.

5. Ruderman N, Chisholm D, Pi-Sunyer X. The metabolically obese, normal weight individual revisited. Diabetes 1998; 47: 699-713.

6. Pajunen p, Kotronen A, Korpi-Hyövälti E, Keinänen-Kiukaanniemi S, Oksa H, Niskanen L, Saaristo T, Saltevo J, Sundvall J, Vanhala M, Uusitupa M, Peltonen M. Obesity phenotypes in the general population: the FIN-D2D Survey. BMC Public Health 2011; 11: 754.

7. Goday A, Eva C, Vazquez LA, Caveda E, Margallo T, Catalina-Romero C, Reviriego J. Prevalence and clinical characteristics of metabolically healthy obese individuals and other obese/non-obese metabolic phenotypes in a working population: results from the Icaria study. BMC Public Health 2016; 16: 248.

8. Wildman RP, Muntner P, Reynolds K. The obese without cardiometabolic risk factor clustering and the normal weight with cardiometabolic risk factor clustering: prevalence and correlates of 2 phenotypes among the US population (NHANES 1999-2004). Arch Int Med 2008; 168(15): 1617-1624. 